\title{
Thymidylate synthase locus LOH in combination with genotype has prognostic and predictive significance in colorectal cancer
}

\author{
MASANORI KOTAKE ${ }^{1-4}$, HIROYUKI BANDO ${ }^{2}$, MAMI KANEKO ${ }^{1,2,4}$, HIROFUMI TAKEMURA ${ }^{1}$, \\ TOSHINARI MINAMOTO ${ }^{4}$ and KAZUYUKI KAWAKAMI ${ }^{4,5}$
}

\begin{abstract}
${ }^{1}$ Department of General and Cardiothoracic Surgery, Graduate School of Medical Science, Kanazawa University, Kanazawa, Ishikawa 920-8641; ${ }^{2}$ Department of Gastrointestinal Surgery, Ishikawa Prefectural Central Hospital, Kanazawa, Ishikawa 920-8530; ${ }^{3}$ Department of Surgery, Kouseiren Takaoka Hospital, Takaoka, Toyama 933-8555;

${ }^{4}$ Division of Translational and Clinical Oncology, Cancer Research Institute, Kanazawa University, Kanazawa, Ishikawa 920-0934; ${ }^{5}$ Department of Chemotherapy and Palliative Care, Tokyo Women's Medical University,
\end{abstract} Tokyo 162-8666, Japan

Received September 29, 2020; Accepted July 22, 2021

DOI: $10.3892 /$ mco.2021.2398

\begin{abstract}
The aim of the current study was to investigate the prognostic and predictive significance of polymorphisms in the thymidylate synthase (TS) gene, alongside the loss of heterozygocity ( $\mathrm{LOH})$ at this gene locus in patients with colorectal cancer. Genotyping was carried out for a variable number tandem repeat (VNTR) polymorphism in the TS 5'-untranslated region, a G/C single nucleotide polymorphism (SNP) located within this VNTR, and for TS LOH status in 246 colorectal cancer and paired normal DNA samples. The results were analyzed in relation to clinicopathological features, including the prognostic and predictive significance of TS genotype in patients who underwent curative surgery. Complete VNTR, SNP and LOH information for TS was obtained in 226 cases. No significant associations were observed between normal tissue TS genotype status and clinicopathological features. LOH of TS was observed in 58\% of tumor samples and was associated with poor prognosis independently of clinical stage.
\end{abstract}

Correspondence to: Dr Masanori Kotake, Department of Surgery, Kouseiren Takaoka Hospital, 5-10 Eiraku-cho, Takaoka, Toyama 933-8555, Japan

E-mail: kotake628@gmail.com

Professor Toshinari Minamoto, Division of Translational and Clinical Oncology, Cancer Research Institute, Kanazawa University, 13-1 Takara-machi, Kanazawa, Ishikawa 920-0934, Japan

E-mail: minamoto@staff.kanazawa-u.ac.jp

Abbreviations: CRC, colorectal cancer; LOH, loss of heterozygosity; PE, primer extension; RFLP, restriction fragment length polymorphism; SNP, single nucleotide polymorphism; TS, thymidylate synthase; 5'-UTR, 5'-untranslated region; VNTR, variable number tandem repeat

Key words: 5-fluorouracil, colorectal cancer, genetic polymorphism, loss of heterozygocity, thymidylate synthase
Cases exhibiting TS LOH were classified into the three groups of $2 \mathrm{R} / \mathrm{loss}, 3 \mathrm{G} / \mathrm{loss}$ and $3 \mathrm{C} / \mathrm{loss}$. Patients with $3 \mathrm{C} /$ loss genotype status had poor outcomes when treated by surgery alone, but their survival was similar to patients with other genotypes following Fluorouracil (5-FU)-based adjuvant chemotherapy. The results suggested that $\mathrm{LOH}$ of the TS locus may be a significant prognostic factor in colorectal cancer, with the genotype of the residual allele also demonstrating an influence on prognosis. In conclusion, LOH status should be considered when TS genotype is explored as a potential prognostic and predictive marker for 5-FU-based adjuvant chemotherapy in colorectal cancer.

\section{Introdution}

Colorectal cancer (CRC) is one of the most common malignancies worldwide. Although most localized cases of the disease are treated surgically, a considerable number of patients experience disease recurrence. Adjuvant chemotherapy after curative surgery has been shown to reduce the recurrence rate and therefore all CRC patients with clinical stage III disease are recommended to receive adjuvant chemotherapy (1), even though only a proportion of these derive a benefit. Patients with clinical stage II disease are mostly treated with surgery alone, however some may benefit from adjuvant therapy because of a high risk of recurrence $(2,3)$. To maximize the efficacy of adjuvant chemotherapy, accurate predictive markers are needed to select patients who will benefit most from treatment.

5-FU-based chemotherapy is the current standard of care for adjuvant therapy following surgical treatment of CRC (4). Thymidylate synthase (TS) is a target enzyme for 5-FU (5), leading to extensive studies of TS mRNA expression (6), TS protein expression $(7,8)$ and TS gene polymorphisms $(9,10)$ as potential predictive factors for the efficacy of 5-FU-based adjuvant chemotherapy. Currently however, no information regarding TS status is recommended for routine clinical use in the selection of patients to receive 5-FU-based chemotherapy (11). 
TS shows unique genetic variants comprising a variable number of tandem repeat (VNTR) and a single nucleotide polymorphism (SNP) in its 5' untranslated region (5'UTR) (12-14). These may be predictive markers for 5-FU efficacy and for adverse events from this treatment. We previously reported that VNTR and SNP can give rise to four TS allele types: $2 \mathrm{G}, 2 \mathrm{C}, 3 \mathrm{G}$ and $3 \mathrm{C}$. These may affect the translational activity of TS mRNA, thus influencing TS protein expression and therefore constitute a marker for the efficacy of 5-FU-based adjuvant chemotherapy (14-16). In addition to these four allele types, other rarer alleles comprising more than three repeats and novel SNPs in the $2 \mathrm{R}$ allele have also been reported (17), thus giving rise to a larger number of allele types. Furthermore, we observed that frequent loss of heterozygosity ( $\mathrm{LOH}$ ) of the TS locus in tumors can affect the genotype, thereby indirectly influencing the TS expression level in tumors $(18,19)$. This potential change in genotype status due to LOH should be considered in studies of the TS genotype as a predictive marker. The status of VNTR, SNPs in both $2 \mathrm{R}$ and $3 \mathrm{R}$, and $\mathrm{LOH}$ must all be evaluated before TS genotype information can be introduced into the clinical setting.

In this study, we analyzed the TS VNTR, the SNPs in both the $2 \mathrm{R}$ and $3 \mathrm{R}$ alleles, as well as the $\mathrm{LOH}$ status of the TS locus in order to explore their potential significance as prognostic and predictive markers of 5-FU-based adjuvant chemotherapy in CRC.

\section{Materials and methods}

Patient cohort and DNA isolation. Matched tumor and normal tissue samples were obtained following surgical resection for primary colorectal adenocarcinoma in 246 patients. The patients were all Japanese and comprised 146 males and 100 females, ranging in age from 33 to 93 years (mean age 66.0 years). The resected tissues were fixed in formalin and embedded in paraffin followed by H\&E staining and histological diagnosis. Tumor tissue was dissected manually from $10 \mu \mathrm{m}$ sections of formalin-fixed, paraffin-embeded tissue blocks. After deparaffinization using xylene and ethanol, genomic DNA was isolated using a QIAamp DNA FFPE Tissue Kit (QIAGEN, Hilden, Germany) following the protocol provided by the manufacturer. Approval for this project was obtained from the Kanazawa University Genome/Gene Analysis Research Ethics Committee.

Genotyping of TS VNTR and SNP. TS genotypes for the VNTR and the SNP in the 3R allele were determined by PCR and PCR-restriction fragment length polymorphism (RFLP) using the forward primer TS25: 5'-AGGCGCGCG GAAGGGGTCCT-3' and reverse primer TS18: 5'-TCCGAG CCGGCCACAGGCAT-3' as described previously (14) with a modification of PCR conditions. PCR with the genomic DNA template was performed in reaction mixtures containing $1 \mathrm{X}$ TaKaRa HS Taq buffer (TaKaRa Bio, Otsu, Japan), $200 \mu \mathrm{M}$ deoxyribonucleoside triphosphates, $500 \mathrm{nM}$ of each primer, 0.5 unit of TaKaRa HS Taq DNA polymerase (TaKaRa Bio) and $100 \mathrm{ng}$ of genomic DNA. The cycling conditions were: one cycle at $95^{\circ} \mathrm{C}$ for $3 \mathrm{~min}, 35$ cycles at $98^{\circ} \mathrm{C}$ for $10 \mathrm{sec}$ and $68^{\circ} \mathrm{C}$ for $60 \mathrm{sec}$, with a final extension at $72^{\circ} \mathrm{C}$ for $5 \mathrm{~min}$. Aliquots of amplified fragments were separated on 3\% agarose gels to determine the TS VNTR genotype.

Samples showing the $2 \mathrm{R} / 3 \mathrm{R}$ or $3 \mathrm{R} / 3 \mathrm{R}$ genotypes were analyzed further for the $\mathrm{G} / \mathrm{C}$ polymorphism in the $3 \mathrm{R}$ allele by using the RFLP method. HaeIII digestion of the 3R fragment produced 66-, 37-, 28- and 10-bp bands for the 3G allele, and 94-, 37- and 10-bp bands for the 3C allele after separation on $3 \%$ agarose gels. For samples with $2 \mathrm{R} / 2 \mathrm{R}$ or $2 \mathrm{R} / 3 \mathrm{R}$ genotypes, the $\mathrm{G} / \mathrm{C}$ polymorphism in the $2 \mathrm{R}$ allele was determined by PCR-PERFLP method, consisting of PCR followed by primer extension (PE) and RFLP analysis with HaeIII digestion. The PCR reaction was performed using reverse primer TS21: 5'-CAGCTCCGAGCCGGCCACAG-3' instead of TS18. Five microliters of PCR product was mixed with extension primer TS105: 5'-TCCGAGCCAGCCACAGGCAT-3' labeled with fluorescein 5'-isothiocyanate to a total volume of $7.5 \mu \mathrm{l}$. The mixture was denatured for $5 \mathrm{~min}$ at $98^{\circ} \mathrm{C}$, annealed for $10 \mathrm{~min}$ at room temperature and then combined with $2.5 \mu \mathrm{l}$ of $\mathrm{PE}$ reaction mixture containing 0.5 unit of Vent (exo-) DNA polymerase (New England BioLabs, Ipswich, MA), $200 \mu \mathrm{M}$ deoxyribonucleoside triphosphates, $1 \mathrm{X}$ ThermoPol Reaction Buffer provided by the manufacturer, followed by incubation for $10 \mathrm{~min}$ at $72^{\circ} \mathrm{C}$. The product of primer extension was digested with HaeIII, separated on $3 \%$ agarose gels and visualized with the Typhoon fluoroimager. The $2 \mathrm{G}$ allele produced a $48 \mathrm{bp}$ fragment and the $2 \mathrm{C}$ allele a $76 \mathrm{bp}$ fragment with the fluorescein 5 '-isothiocyanate label. The TS genotype was thus classified into $2 \mathrm{G} / 2 \mathrm{G}, 2 \mathrm{G} / 2 \mathrm{C}, 2 \mathrm{C} / 2 \mathrm{C}, 2 \mathrm{G} / 3 \mathrm{G}, 2 \mathrm{G} / 3 \mathrm{C}$, $2 \mathrm{C} / 3 \mathrm{G}, 2 \mathrm{C} / 3 \mathrm{C}, 3 \mathrm{G} / 3 \mathrm{G}, 3 \mathrm{G} / 3 \mathrm{C}$, or $3 \mathrm{C} / 3 \mathrm{C}$ by comprehensive genotyping of the VNTR and SNP in the TS 5'UTR. Analyses were performed at least twice to confirm the genotype.

LOH analysis. LOH of the TS locus was determined in three distinct ways depending on the TS genotype observed in the normal tissue. The G/C SNP in the $2 \mathrm{R}$ allele was not taken into consideration for $\mathrm{LOH}$ analyses. Samples that were $2 \mathrm{R} / 3 \mathrm{G}$ or $2 \mathrm{R} / 3 \mathrm{C}$ were analyzed by PCR followed by separation on Spreadex gel (Elchrom Scientific, Cham, Switzerland). Samples that were $2 \mathrm{R} / 2 \mathrm{R}, 3 \mathrm{G} / 3 \mathrm{G}$ or $3 \mathrm{C} / 3 \mathrm{C}$ were evaluated for $\mathrm{LOH}$ using the microsatellite marker D18S59, as described previously (18). Samples that were $3 \mathrm{G} / 3 \mathrm{C}$ were analyzed using the PCR-PERFLP method, as described above for the SNP genotyping method with the $2 \mathrm{R}$ allele. PCR-PERFLP avoids interference due to heteroduplex formation, thereby allowing the exact allele ratio to be determined. The $3 \mathrm{G}$ allele produced a 76 bp fragment and the 3C allele a 104 bp fragment with PCR-PERFLP, as visualized by Typhoon fluoroimaging. The image was analyzed using ImageQuant software and the relative ratio between $3 \mathrm{G}$ and $3 \mathrm{C}$ alleles in tumor DNA was normalized using the ratio measured in the corresponding normal tissue DNA sample. LOH was defined as either the complete absence of one allele, or a decrease in intensity of one allele by at least $50 \%$. LOH of $18 \mathrm{q}$ was analyzed using the microsatellite markers D18S58, D18S61 and D18S64. Forward primers were labeled with fluorescein 5'-isothiocyanate and the same method as for microsatellite marker D18S59 was used.

Statistical analysis. Relationships between variables were analyzed by Chi-square analysis or the Scheffe post-hoc test used following ANOVA. The cumulative survival rate was 
estimated using the Kaplan-Meier method and statistical significance was assessed by the log-rank test. Cox regression modelling was used for multivariate analysis. P-values less than 0.05 were considered significant.

\section{Results}

Genotype analysis in normal tissue samples. We previously investigated and compared the TS genotypes between matched normal and tumor tissues from 151 patients with colorectal cancer. The results suggest that frequent $\mathrm{LOH}$ of the TS locus detected in the tumors affect the functional TS genotyping (18). Therefore, in the present study, TS genotyping was carried out on DNA from normal tissue rather than from tumor samples in order to avoid possible artifacts from LOH. The VNTR genotype distribution amongst the 246 cases was: $2 R / 2 R(n=9)$, $2 R / 3 R(n=70), 3 R / 3 R(n=162)$ and $3 R / 5 R(n=5)$. Because the $5 R$ allele is rare and analysis of the G/C SNP in the repeat component is difficult, the 5 cases with $3 R / 5 R$ genotype were excluded. The remaining 241 cases of $2 R / 2 R, 2 R / 3 R$ and $3 R / 3 R$ VNTR genotype cases were then screened for the G/C SNP in both the $2 \mathrm{R}$ and $3 \mathrm{R}$ alleles (Fig. 1). The combined VNTR and SNP genotype frequencies were: $2 \mathrm{G} / 2 \mathrm{C}(\mathrm{n}=1), 2 \mathrm{C} / 2 \mathrm{C}$ $(\mathrm{n}=8), 2 \mathrm{G} / 3 \mathrm{G}(\mathrm{n}=3), 2 \mathrm{G} / 3 \mathrm{C}(\mathrm{n}=3), 2 \mathrm{C} / 3 \mathrm{G}(\mathrm{n}=25), 2 \mathrm{C} / 3 \mathrm{C}(\mathrm{n}=39)$, $3 \mathrm{G} / 3 \mathrm{G}(\mathrm{n}=48), 3 \mathrm{G} / 3 \mathrm{C}(\mathrm{n}=81)$ and $3 \mathrm{C} / 3 \mathrm{C}(\mathrm{n}=33)$. The previously reported $2 \mathrm{R}$ allele with a $\mathrm{G} \rightarrow \mathrm{C}$ SNP located in the first tandem repeat (17) was not found in our subjects. This should have resulted in a $121 \mathrm{bp}$ fragment following PCR-PERFLP and gel electrophoresis (Fig. 1B). The $2 \mathrm{G}$ allele was quite rare in our population and showed no significant associations with any clinicopathological variable or with clinical course (data not shown). Therefore, SNP information for the $2 \mathrm{R}$ allele was not considered in further analysis. The distribution of clinicopathological features according to TS VNTR/SNP genotype are shown in Table I. As reported previously (14), the 3G allele was less frequent in females $(\mathrm{P}=0.04$, chi-square test) (male $\mathrm{G}=115$, female $\mathrm{G}=62$, male $\mathrm{C}=79$, female $\mathrm{C}=68$ ). No significant associations were apparent between the normal tissue TS genotype and any other clinicopathological feature.

$\mathrm{LOH}$ and the residual TS allele in colorectal cancer. The appropriate method for $\mathrm{LOH}$ analysis was selected according to the genotype found in the normal tissue, as shown in Fig. 2. The genotype frequency according to LOH status is also shown in Fig. 2. A novel method for $\mathrm{LOH}$ analysis of the $3 \mathrm{G} / 3 \mathrm{C}$ genotype was employed in this study and involved the use of PCR-PERFLP to avoid interference with heteroduplex product (Fig. 3). Fifteen cases could not be evaluated for LOH status because both the TS genotype and the D18S59 microsatellite marker were homozygous. Therefore, complete TS VNTR/SNP genotype information together with tumor $\mathrm{LOH}$ status was available for 226 patients. The frequencies were: $2 R / 2 R(n=3), 2 R / 3 G(n=11), 2 R / 3 C(n=20), 3 G / 3 G$ $(\mathrm{n}=13), 3 \mathrm{G} / 3 \mathrm{C}(\mathrm{n}=34), 3 \mathrm{C} / 3 \mathrm{C}(\mathrm{n}=14), 2 \mathrm{R} /$ loss $(\mathrm{n}=26), 3 \mathrm{G} / \mathrm{loss}$ $(n=58)$ and $3 \mathrm{C} /$ loss $(n=47)$. The overall frequency of $\mathrm{LOH}$ for the TS locus was $58.0 \%(131 / 226)$. Lossed alleles were evenly distributed between $2 \mathrm{R}(\mathrm{n}=23), 3 \mathrm{G}(\mathrm{n}=58)$ and $3 \mathrm{C}(\mathrm{n}=50)$. Associations between LOH status and clinicopathological features are shown in Table II. The absence of LOH for TS was significantly associated with proximal tumor location
A

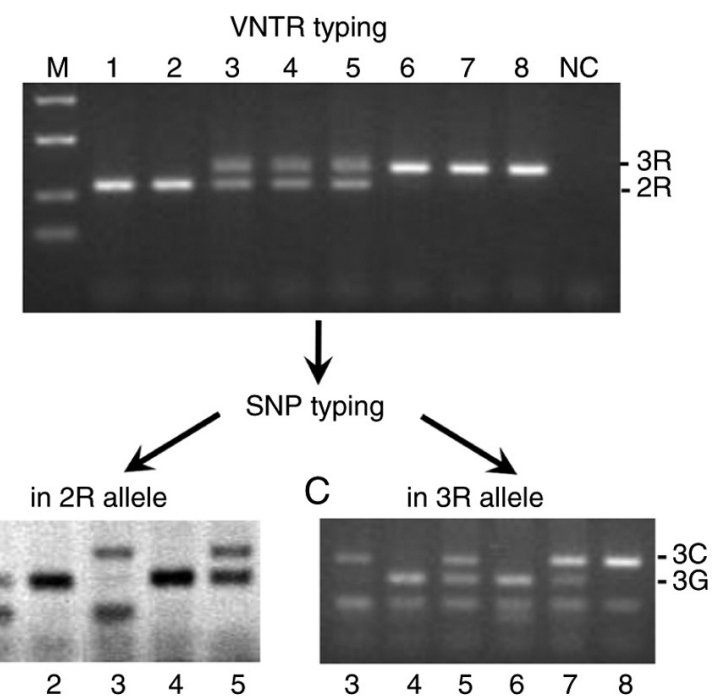

Figure 1. TS VNTR and SNP analysis. (A) VNTR analysis using PCR amplification and separation of products on a 3\% agarose gel. (B) SNP analysis in the $2 \mathrm{R}$ allele using PERFLP followed by separation on a $3 \%$ agarose gel and scanning with a fluoroimager. (C) SNP analysis in the 3R allele by RFLP and separation on a $3 \%$ agarose gel. The DNA fragments stained using ethidium bromide are displayed as white pixels on a black background, whereas those labeled by fluoresein are displayed as black pixels on a white background. The numbers on these panels indicate the same samples being analyzed. The genotypes are as follows: $1,2 \mathrm{G} / 2 \mathrm{C} ; 2,2 \mathrm{C} / 2 \mathrm{C} ; 3,2 \mathrm{G} / 3 \mathrm{C} ; 4,2 \mathrm{C} / 3 \mathrm{G}$; $5,2 \mathrm{C} / 3 \mathrm{C} ; 6,3 \mathrm{G} / 3 \mathrm{G} ; 7,3 \mathrm{G} / 3 \mathrm{C}$; and $8,3 \mathrm{C} / 3 \mathrm{C}$. $\mathrm{M}$ indicates the size marker $(50,100,200$ and $300 \mathrm{bp})$ and $\mathrm{NC}$ indicates that there was no template control. TS, thymidylate synthase gene; VNTR, variable number tandem repeat; SNP, single nucleotide polymorphisml RFLP, restriction fragment length polymorphism.

and with mucinous histology. No other statistically significant associations were observed.

Patient prognosis and TS LOH status. A number of studies have reported that $\mathrm{LOH}$ at a given gene locus is associated with poor prognosis. We therefore analyzed the prognostic significance of TS LOH status before examining the prognostic role of the TS genotype. This was performed for 153 patients with clinical stage II or III disease who underwent curative surgery and where clinical information including long term follow-up and use of adjuvant therapy was available. Of these patients, 90 (59\%) showed TS LOH and had significantly shorter survival $(\mathrm{P}=0.0005)$ compared to patients with no $\mathrm{LOH}(\mathrm{n}=63$; Fig. 4). We also compared the frequency of LOH between the tumors at clinical stage II and III by Chi-square test. We found no statistical difference in frequency of LOH between the two groups of tumors $(\mathrm{P}=0.22)$. Multivariate analysis with the parameters listed in Table III demonstrated that TS LOH status, receipt of adjuvant chemotherapy and clinical stage were independent prognostic factors in this patient cohort.

TS LOH is often accompanied by $18 q \mathrm{LOH}$. TS is located on 18p11.32. Earlier studies reported that $18 \mathrm{q} \mathrm{LOH}$ was associated with poor prognosis in CRC $(20,21)$. Thus, we analyzed the relation between TS LOH and 18q LOH in 41 randomly selected samples comprising 15 cases with no TS LOH and 26 cases with TS LOH. Three microsatellite markers (D18S58, D18S61, D18S64) located at 18q22.3, 18q22.2 and 18q21.32, respectively, were used to determine 18q LOH. Fig. 5 shows the TS LOH 
Table I. Thymidylate synthase genotype and clinicopathological features.

\begin{tabular}{|c|c|c|c|c|c|c|}
\hline \multirow[b]{2}{*}{ Parameter } & \multicolumn{3}{|c|}{$2 \mathrm{R} / 3 \mathrm{R}$} & \multicolumn{3}{|c|}{$3 \mathrm{R} / 3 \mathrm{R}$} \\
\hline & $2 \mathrm{R} / 3 \mathrm{R}$ & $2 \mathrm{R} / 3 \mathrm{G}$ & $2 \mathrm{R} / 3 \mathrm{C}$ & $3 \mathrm{G} / 3 \mathrm{G}$ & $3 \mathrm{G} / 3 \mathrm{C}$ & $3 \mathrm{C} / 3 \mathrm{C}$ \\
\hline Total & 9 & 28 & 42 & 48 & 81 & 33 \\
\hline \multicolumn{7}{|l|}{ Sex } \\
\hline Male & 5 & 18 & 23 & 37 & 41 & 19 \\
\hline Female & 4 & 10 & 19 & 11 & 40 & 14 \\
\hline \multicolumn{7}{|l|}{ Age (years) } \\
\hline Mean & 63.3 & 70.4 & 63.7 & 66.3 & 65.5 & 66.3 \\
\hline SD & 15.8 & 11.1 & 11.2 & 13.2 & 11.1 & 13.5 \\
\hline \multicolumn{7}{|l|}{ Stage } \\
\hline I & 1 & 4 & 6 & 3 & 7 & 3 \\
\hline II & 5 & 5 & 17 & 18 & 32 & 10 \\
\hline III & 3 & 13 & 17 & 14 & 27 & 13 \\
\hline IV & 0 & 6 & 2 & 13 & 15 & 7 \\
\hline \multicolumn{7}{|l|}{ Tumor site } \\
\hline Proximal & 2 & 9 & 12 & 19 & 32 & 9 \\
\hline Distal & 7 & 19 & 30 & 29 & 49 & 24 \\
\hline \multicolumn{7}{|l|}{ Pathology } \\
\hline Tub1 & 7 & 8 & 17 & 21 & 39 & 13 \\
\hline Tub2 & 1 & 17 & 21 & 23 & 36 & 16 \\
\hline Muc & 1 & 0 & 1 & 2 & 1 & 2 \\
\hline Por & 0 & 3 & 3 & 2 & 5 & 2 \\
\hline
\end{tabular}

Muc, mucinous adenocarcinoma; Por, poorly differentiated adenocarcinoma; Tub1, well differentiated tubular adenocarcinoma; Tub2, moderately differentiated tubular adenocarcinoma.

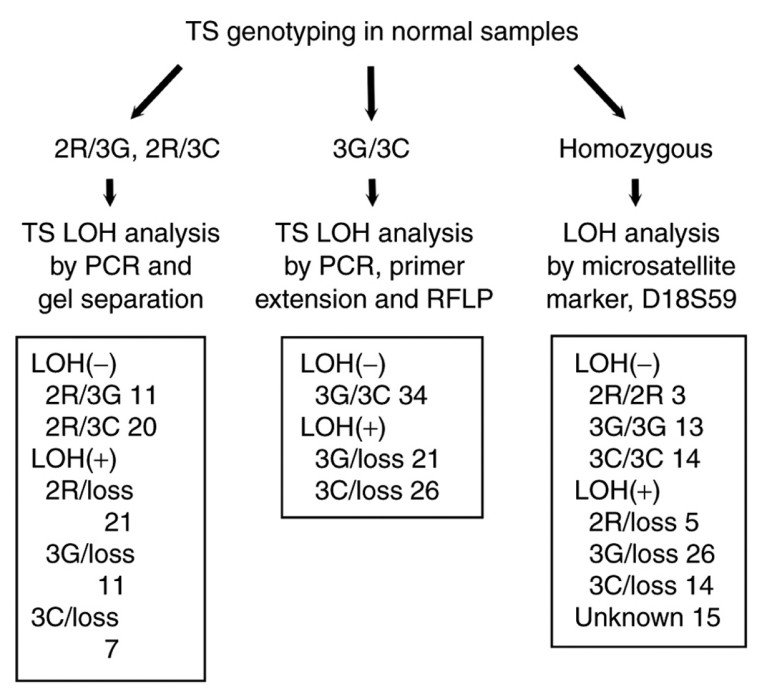

Figure 2. Flow chart of LOH analysis and the frequency of TS genotypes. The LOH status of tumors was analyzed according to the TS genotype in normal sample. The TS genotype frequency in relation to $\mathrm{LOH}$ status is shown in the box. LOH, loss of heterozygocity; TS, thymidylate synthase gene; RFLP, restriction fragment length polymorphism.

status as well as that of each $18 \mathrm{q}$ microsatellite marker. Whenever LOH was observed at the TS gene locus, it was also consistently present at other $18 \mathrm{q}$ loci. On the other hand, three tumors (from

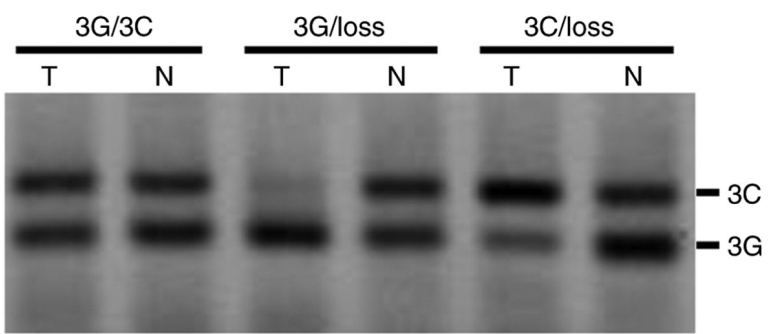

Figure 3. Representative cases of LOH analysis in 3G/3C genotype samples. The PCR-primer extention restriction fragment length polymorphism method detailed in the materials and methods was used to avoid interference by the heteroduplex product. The image of DNA fragments labeled by fluoresein are displayed as black pixels on a white background. Thymidylate synthase genotype and LOH status is indicated on the top of matched $\mathrm{T}$ and $\mathrm{N}$ lanes. $\mathrm{LOH}$, loss of heterozygocity; T, tumor; N, normal.

patients no. 4, 13 and 16 shown in Fig. 5) showed LOH at one or more $18 \mathrm{q}$ microsatellite markers in the absence of LOH at TS. In these tumors, $\mathrm{LOH}$ was not observed for all three markers, indicating the chromosomal loss occurred in a relatively small area of $18 \mathrm{q}$. These results suggest that $\mathrm{LOH}$ at TS and $18 \mathrm{q}$ are simultaneous events in most $\mathrm{CRC}$, although a few tumors have small areas of $\mathrm{LOH}$ at $18 \mathrm{q}$ without allelic loss at the TS locus.

TS genotype as a prognostic and predictive factor in tumors with $\mathrm{LOH}$. Since TS LOH was a strong prognostic factor (Fig. 4) 
Table II. Loss of heterozygosity of the thymidylate synthase locus and clinicopathological features.

\begin{tabular}{lccc}
\hline Parameter & No LOH & LOH & P-value \\
\hline Total & 95 & 131 & \\
Sex & & & 0.63 \\
Male & 55 & 80 & \\
Female & 40 & 51 & \\
Age (years) & & & 0.16 \\
Mean & 64.6 & 66.9 & \\
SD & 12.9 & 11.3 & \\
Stage & & & \\
I & 11 & 12 & \\
II & 30 & 52 & \\
III & 39 & 46 & \\
IV & 15 & 21 & \\
Tumor site & & & \\
Proximal & 48 & 30.038 \\
Distal & 47 & 101 & \\
Pathology & & 69001 \\
Tub1 & 42 & 65 & \\
Tub2 & 39 & 69 & \\
Muc & 6 & 6 & \\
Por & & & \\
\hline
\end{tabular}

Muc, mucinous adenocarcinoma; Por, poorly differentiated adenocarcinoma; SD, standard deviation; Tub1, well differentiated tubular adenocarcinoma; Tub2, moderately differentiated tubular adenocarcinoma.

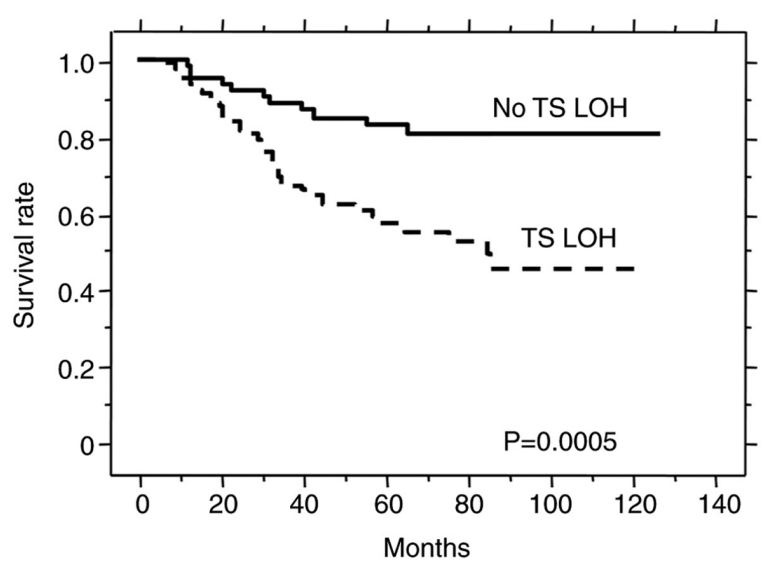

Figure 4. Overall survival of patients with curatively resected colorectal cancer according to their TS LOH status. TS, thymidylate synthase gene; $\mathrm{LOH}$, loss of heterozygocity.

and also influences the TS genotype observed in tumors, we explored the role of TS genotype separately in patient groups stratified according to their LOH status. In patients without TS LOH $(n=63)$, the tumor genotype is identical to that found in normal tissue. These patients were classified into 6 groups: $2 R / 2 R(n=2), 2 R / 3 G(n=6), 2 R / 3 C \quad(n=13), 3 G / 3 G(n=10)$, $3 \mathrm{G} / 3 \mathrm{C}(\mathrm{n}=23)$ and $3 \mathrm{C} / 3 \mathrm{C}(\mathrm{n}=9)$. The relatively small number
Table III. Multivariate analysis of prognostic factors.

\begin{tabular}{|c|c|c|c|}
\hline Variable & Hazard ratio & $95 \% \mathrm{CI}$ & $\mathrm{P}$-value \\
\hline \multicolumn{4}{|l|}{ TS LOH } \\
\hline Yes vs. no & 3.01 & $1.36-6.64$ & 0.0065 \\
\hline \multicolumn{4}{|c|}{ Adjuvant chemotherapy } \\
\hline Yes vs. no & 0.53 & $0.28-0.98$ & 0.045 \\
\hline \multicolumn{4}{|l|}{ Sex } \\
\hline Male vs. female & 1.22 & $0.66-2.26$ & 0.53 \\
\hline \multicolumn{4}{|l|}{ Age } \\
\hline$\geq 66$ vs. $<66$ & 1.15 & $0.62-2.16$ & 0.66 \\
\hline \multicolumn{4}{|l|}{ Stage } \\
\hline II vs. III & 0.53 & $0.29-0.96$ & 0.039 \\
\hline \multicolumn{4}{|l|}{ Tumor site } \\
\hline Proximal vs. distal & 0.63 & $0.28-1.43$ & 0.27 \\
\hline \multicolumn{4}{|l|}{ Pathology } \\
\hline Tub2 vs. Tub1 & 1.27 & $0.368-2.38$ & 0.45 \\
\hline Muc vs. Tub1 & 2.42 & $0.50-11.7$ & 0.27 \\
\hline Por vs. Tub1 & 0.53 & $0.068-4.21$ & 0.55 \\
\hline
\end{tabular}

CI, confidence interval; LOH, loss of heterozygosity; M, mucinous adenocarcinoma; Por, poorly differentiated adenocarcinoma; TS, thymidylate synthase; Tub1, well differentiated tubular adenocarcinoma; Tub2, moderately differentiated tubular adenocarcinoma.

of cases for each genotype prevented analysis of the prognostic value of these groups. When the genotypes were grouped into L-type $(2 \mathrm{R} / 2 \mathrm{R}, 2 \mathrm{R} / 3 \mathrm{C}, 3 \mathrm{C} / 3 \mathrm{C} ; \mathrm{n}=24)$ and H-type $(2 \mathrm{R} / 3 \mathrm{G}$, $3 \mathrm{G} / 3 \mathrm{C}, 3 \mathrm{G} / 3 \mathrm{G} ; \mathrm{n}=39$ ) according to criteria from our previous report (14), no prognostic significance was observed in the overall patient group, in patients treated by surgery alone, or in patients treated with adjuvant chemotherapy (data not shown).

In patients with TS LOH ( $n=90)$, the tumor TS genotypes were: $2 R / \operatorname{loss}(n=20), 3 \mathrm{G} / \operatorname{loss}(n=39)$ and $3 \mathrm{C} / \operatorname{loss}(n=31)$. No prognostic significance was observed for these genotypes in the overall group of patients with TS LOH (Fig. 6A) or in patients who received adjuvant chemotherapy (Fig. 6C). However, the $3 \mathrm{C} /$ loss genotype was associated with significantly shorter survival in patients treated by surgery alone (Fig. 6B). These results suggest that patients with the $3 \mathrm{C} /$ loss genotype have poor prognosis when treated by surgery alone, but may benefit from chemotherapy as observed by the similar survival rate to patients with other genotypes. Indeed, patients with the $3 \mathrm{C} /$ loss genotype who received adjuvant chemotherapy survived significantly longer than those treated by surgery alone (Fig. 6D). Thus, the $3 \mathrm{C} /$ loss genotype appears to be a prognostic marker for poor outcome, as well as a predictive marker for good response to 5-FU-based chemotherapy.

\section{Discussion}

In this report we genotyped TS for VNTR status and for SNPs located within the $2 \mathrm{R}$ and $3 \mathrm{R}$ alleles. We have evaluated the TS locus for LOH in CRC. In agreement with our previous observations, $\mathrm{LOH}$ was quite frequent regardless of the TS 


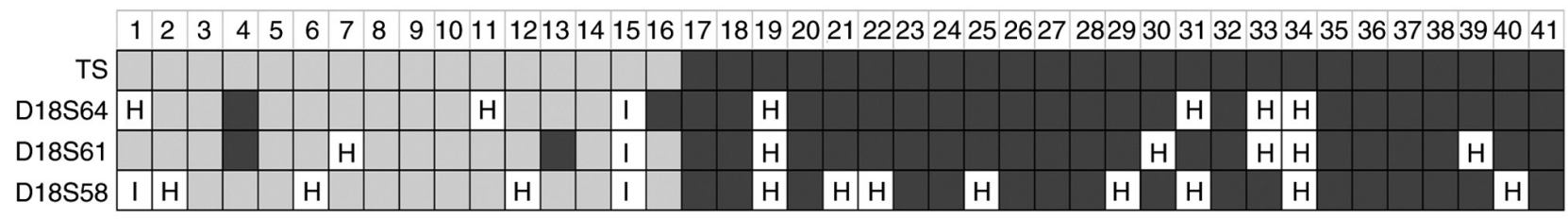

Figure 5. LOH status of the TS locus and of 18q in 41 randomly selected tumor samples. White squares indicate that the LOH status could not be determined either due to microsatellite instability (I) or homozygosity (H). Light grey squares indicate no LOH and dark grey squares indicate LOH. LOH, loss of heterozygocity; TS, thymidylate synthase gene.
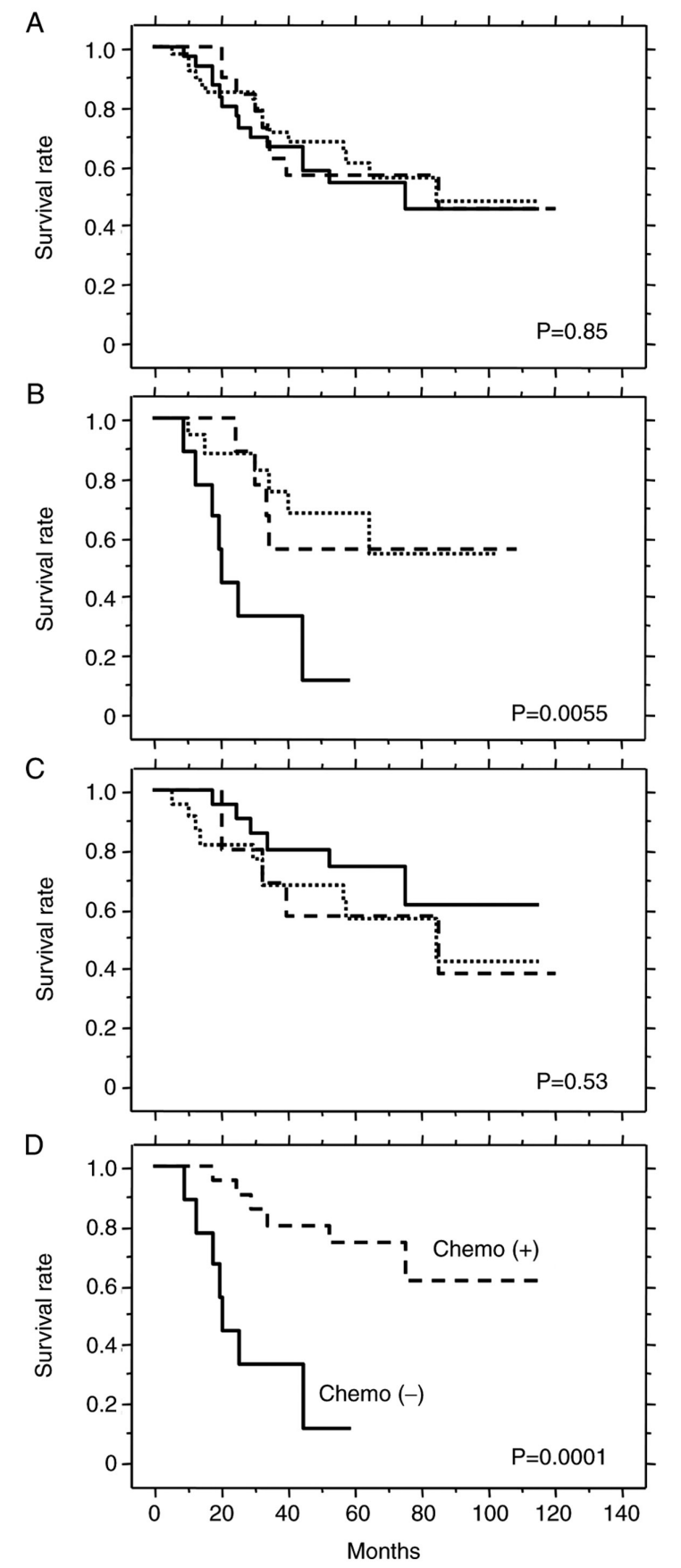

Figure 6. Survival analysis of patients with colorectal cancer exhibiting TS LOH. The survival rates of patients with $2 \mathrm{R} /$ loss (broken line), $3 \mathrm{G} /$ loss (dotted line) and 3C/loss (continuous line) genotype were compared using the Kaplan-Meier method in (A) all patients with TS LOH, (B) patients treated with surgery alone and $(\mathrm{C})$ patients who received adjuvant chemotherapy. (D) Survival of patients with the $3 \mathrm{C} /$ loss genotype who did (broken line) or did not (continous line) receive 5-FU-based adjuvant chemotherapy. TS, thymidylate synthase gene; LOH, loss of heterozygocity; 5-FU, Fluorouracil. genotype $(18,19)$. TS LOH was a prognostic factor for poor survival (Fig. 4), independently of clinical stage and other clinicopathological features. Because of the high frequency of TS LOH (58\%) and also its significant prognostic impact, the TS genotype cannot be combined with $\mathrm{LOH}$ status to give one simple prognostic indicator similar for example to the ' $3 \mathrm{G}$-containing type' we used previously (14). The $3 \mathrm{G} / 3 \mathrm{G}$ and $3 \mathrm{G} /$ loss genotypes are identical in that both have only the $3 \mathrm{G}$ allele, however their prognostic significance is quite different due to the presence of $\mathrm{LOH}$ in the latter. Important prognostic information derived from the $\mathrm{LOH}$ status would be lost if the $3 \mathrm{G} / 3 \mathrm{G}$ and $3 \mathrm{G} /$ loss genotypes were combined into a simple ' $3 \mathrm{G}$ type'.

In exploring the predictive value of a given factor for adjuvant chemotherapy, it is also important to consider its prognostic value in the absence of such treatment. The TS LOH status is essential for the correct use of TS genotype as a prognostic and predictive factor in 5-FU-based adjuvant chemotherapy. The different number of TS genotypes in tumor DNA is another reason to stratify patients according to their TS LOH status. In cases with no $\mathrm{LOH}, 6$ major TS genotypes are observed $(2 \mathrm{R} / 2 \mathrm{R}, 2 \mathrm{R} / 3 \mathrm{G}, 2 \mathrm{R} / 3 \mathrm{C}, 3 \mathrm{G} / 3 \mathrm{G}, 3 \mathrm{G} / 3 \mathrm{C}, 3 \mathrm{C} / 3 \mathrm{C})$ whereas three groups are seen in cases with $\mathrm{LOH}$ (2R/loss, 3G/loss, 3C/loss). Future investigations into the role of TS genotype in the clinical setting will require large patient cohorts so that the $\mathrm{LOH}$ status of TS can also be taken into account.

In the current study we classified patients according to their TS LOH status and then followed by investigating the prognostic and predictive significance of TS genotype. In cases with no LOH, the overall patient group showed relatively good prognosis (Fig. 4) and there were 6 major genotype groups, making it difficult to obtain statistically meaningful results because of the relatively small patient numbers. Moreover, no prognostic or predictive significance was observed when these 6 genotypes were classified into just two groups $(\mathrm{H}$ and $\mathrm{L})$ according to our previous results (14). This indicates that TS genotype is not a useful marker in patients without TS LOH, although study of a larger number of patients is required to confirm this observation.

Cases with TS LOH showed poor prognosis (Fig. 4). These were further classified into three simple TS genotype groups (2R/loss, 3G/loss, 3C/loss) in order to explore their prognostic and predictive values (Fig. 6). The 3C/loss genotype was a marker for poor prognosis in patients treated by surgery alone (Fig. 6B). Furthermore, the 3C/loss genotype also predicted good response to 5-FU-based adjuvant chemotherapy (Fig. 6D). Despite the relatively small number of patients $(n=31)$, this result reached a high level of statistical significance $(\mathrm{P}=0.0001)$. Using an in vitro reporter assay, we previously showed the $3 \mathrm{C}$ allele was associated with lower translational activity compared 
to the $3 \mathrm{G}$ allele (14). Low expression of TS mRNA (6) and of the TS protein (8) in CRC have both been associated with good response to 5-FU-based chemotherapy. The current result showing the TS $3 \mathrm{C} /$ loss genotype is a marker for good response to 5-FU-based adjuvant chemotherapy (Fig. 6D) is therefore consistent with our previous in vitro observations and with the results of Salonga et al (6) and Soong et al (8). Although we cannot explain why this genotype was associated with poor prognosis (Fig. 6B), the result concurs with a previous study showing that low TS expression is a marker of worse prognosis in patients treated by surgery alone (8).

Due to the retrospective nature of this study and the potential for biases, further analyses are required to validate the results, particularly for the TS genotype groups in cases with $\mathrm{LOH}$. The $2 \mathrm{G}$ allele was rare and no $2 \mathrm{R}$ allele with the $\mathrm{G} \rightarrow \mathrm{C}$ SNP in the first tandem repeat was found in our patient cohort, suggesting that SNP typing of the $2 \mathrm{R}$ allele can be omitted in further studies of the Japanese population. However, there is considerable ethnic variation in the allele frequency for $2 \mathrm{R}$ and the incidence is higher in Western populations (22). Therefore, additional analysis of the SNP in the 2R allele may be required for Caucasian populations.

The LOH status of TS was closely associated with $18 \mathrm{q}$ $\mathrm{LOH}$ status, with the latter being reported previously as a prognostic factor in CRC $(23,24)$. The mechanism by which $18 \mathrm{q} \mathrm{LOH}$ is linked to poor prognosis of CRC patients is not known, although the loss of several tumor suppressor genes in this region including DCC, SMAD4 and SMAD22 has been implicated. The current study sheds light on loss of the whole of chromosome 18 as a prognostic factor. $\mathrm{LOH}$ of $18 \mathrm{p}$ and $18 \mathrm{q}$ should be analyzed simultaneously to investigate whether the minimally lost regions on $18 \mathrm{q}$ or the whole allelic loss of chromosome 18 have stronger prognostic significance.

TS LOH is a significant prognostic factor in CRC. Furthermore, the $3 \mathrm{C} /$ loss genotype appears to be prognostic in patients treated by surgery alone and predictive in patients who receive 5-FU-based adjuvant chemotherapy. Since TS $\mathrm{LOH}$ status influences the TS genotype of tumors and also has a significant prognostic role, TS LOH should be incorporated into all future studies of TS genotype, particularly in relation to its predictive value. Stratification of CRC patients into subgroups defined by TS LOH status is therefore essential in obtaining clear evidence for a clinical role of the TS genotype.

\section{Acknowledgements}

The authors would like to thank Dr. Barry Iacopetta (School of Surgery, University of Western Australia) for the critical reading of the manuscript and for providing valuable suggestions.

\section{Funding}

No funding was received.

\section{Availability of data and materials}

The datasets used and/or analyzed during the current study are available from the corresponding author on reasonable request.

\section{Authors' contributions}

MKo and KK conceived and designed the current study. MKo, HB, MKa and KK collected clinical samples. MKo, $\mathrm{HB}, \mathrm{MKa}, \mathrm{HT}$ and KK performed the experiments and analyzed the data. MKo, TM and KK drafted the manuscript. MKo and TM confirmed the authenticity of all the raw data. All authors read and approved the final version of manuscript.

\section{Ethics approval and consent to participate}

The current study was performed in accordance with the Declaration of Helsinki. Since tissues used in this study were obtained from the patients diagnosed between 1999 and 2010, written informed consent was available for most but not all patients. However in accordance with Japanese ethical guidelines and law, the study protocol was reviewed and approved by the Kanazawa University Human Genome/Gene Analysis Research Ethics Committee (approval nos. 181 and 264). Following instruction by the Ethics Committee at approval, all patients were publicly provided with an opportunity to opt-out from registration to the current study. None declined. All samples were anonymized before analysis was performed to guarantee the protection of privacy.

\section{Patient consent for publication}

Not applicable.

\section{Competing interests}

The authors declare that they have no competing interests.

\section{References}

1. NIH consensus conference. Adjuvant therapy for patients with colon and rectal cancer. JAMA 264: 1444-1450, 1990.

2. Benson AB III, Schrag D, Somerfield MR, Cohen AM, Figueredo AT, Flynn PJ, Krzyzanowska MK, Maroun J, McAllister P, Van Cutsem E, et al: American Society of Clinical Oncology recommendations on adjuvant chemotherapy for stage II colon cancer. J Clin Oncol 22: 3408-3419, 2004.

3. Schmoll HJ, Van Cutsem E, Stein A, Valentini V, Glimelius B, Haustermans K, Nordlinger B, van de Velde CJ, Balmana J, Regula J, et al: ESMO Consensus Guidelines for management of patients with colon and rectal cancer. a personalized approach to clinical decision making. Ann Oncol 23: 2479-2516, 2012.

4. Gill S, Loprinzi CL, Sargent DJ, Thomé SD, Alberts SR, Haller DG, Benedetti J, Francini G, Shepherd LE, Francois Seitz J, et al: Pooled analysis of fluorouracil-based adjuvant therapy for stage II and III colon cancer: Who benefits and by how much? J Clin Oncol 22: 1797-1806, 2004.

5. Danenberg PV: Thymidylate synthetase-a target enzyme in cancer chemotherapy. Biochim Biophys Acta 473: 73-92, 1977.

6. Salonga D, Danenberg KD, Johnson M, Metzger R, Groshen S, Tsao-Wei DD, Lenz HJ, Leichman CG, Leichman L, Diasio RB and Danenberg PV: Colorectal tumors responding to 5-fluorouracil have low gene expression levels of dihydropyrimidine dehydrogenase, thymidylate synthase, and thymidine phosphorylase. Clin Cancer Res 6: 1322-1327, 2000.

7. Edler D, Glimelius B, Hallström M, Jakobsen A, Johnston PG, Magnusson I, Ragnhammar P and Blomgren $\mathrm{H}$ : Thymidylate synthase expression in colorectal cancer: A prognostic and predictive marker of benefit from adjuvant fluorouracil-based chemotherapy. J Clin Oncol 20: 1721-1728, 2002. 
8. Soong R, Shah N, Salto-Tellez M, Tai BC, Soo RA, Han HC, Ng SS, Tan WL, Zeps N, Joseph D, et al: Prognostic significance of thymidylate synthase, dihydropyrimidine dehydrogenase and thymidine phosphorylase protein expression in colorectal cancer patients treated with or without 5-fluorouracil-based chemotherapy. Ann Oncol 19: 915-919, 2008.

9. Iacopetta B, Grieu F, Joseph D and Elsaleh H: A polymorphism in the enhancer region of the thymidylate synthase promoter influences the survival of colorectal cancer patients treated with 5-fluorouracil. Br J Cancer 85: 827-830, 2001.

10. Tsuji T, Hidaka S, Sawai T, Nakagoe T, Yano H, Haseba M, Komatsu H, Shindou H, Fukuoka H, Yoshinaga M, et al: Polymorphism in the thymidylate synthase promoter enhancer region is not an efficacious marker for tumor sensitivity to 5-fluorouracil-based oral adjuvant chemotherapy in colorectal cancer. Clin Cancer Res 9: 3700-3704, 2003.

11. Scartozzi M, Maccaroni E, Giampieri R, Pistelli M, Bittoni A Del Prete M, Berardi R and Cascinu S: 5-Fluorouracil pharmacogenomics: Still rocking after all these years? Pharmacogenomics 12: 251-265, 2011.

12. Horie N, Aiba H, Oguro K, Hojo H and Takeishi K: Functional analysis and DNA polymorphism of the tandemly repeated sequences in the 5'-terminal regulatory region of the human gene for thymidylate synthase. Cell Struct Funct 20: 191-197, 1995.

13. Mandola MV, Stoehlmacher J, Muller-Weeks S, Cesarone G, Yu MC, Lenz HJ and Ladner RD: A novel single nucleotide polymorphism within the 5'tandem repeat polymorphism of the thymidylate synthase gene abolishes USF-1 binding and alters transcriptional activity. Cancer Res 63: 2898-2904, 2003.

14. Kawakami $\mathrm{K}$ and Watanabe G: Identification and functional analysis of single nucleotide polymorphism in the tandem repeat sequence of thymidylate synthase gene. Cancer Res 63 : 6004-6007, 2003

15. Kawakami K, Omura K, Kanehira E and Watanabe Y: Polymorphic tandem repeats in the thymidylate synthase gene is associated with its protein expression in human gastrointestinal cancers. Anticancer Res 19: 3249-3252, 1999.

16. Kawakami K, Salonga D, Park JM, Danenberg KD, Uetake H, Brabender J, Omura K, Watanabe G and Danenberg PV: Different lengths of a polymorphic repeat sequence in the thymidylate synthase gene affect translational efficiency but not its gene expression. Clin Cancer Res 7: 4096-4101, 2001
17. Lincz LF, Scorgie FE, Garg MB and Ackland SP: Identification of a novel single nucleotide polymorphism in the first tandem repeat sequence of the thymidylate synthase $2 \mathrm{R}$ allele. Int J Cancer 120: 1930-1934, 2007.

18. Kawakami K, Ishida Y, Danenberg KD, Omura K, Watanabe G and Danenberg PV: Functional polymorphism of the thymidylate synthase gene in colorectal cancer accompanied by frequent loss of heterozygosity. Jpn J Cancer Res 93: 1221-1229, 2002.

19. Uchida K, Hayashi K, Kawakami K, Schneider S, Yochim JM, Kuramochi H, Takasaki K, Danenberg KD and Danenberg PV: Loss of heterozygosity at the thymidylate synthase (TS) locus on chromosome 18 affects tumor response and survival in individuals heterozygous for a 28-bp polymorphism in the TS gene. Clin Cancer Res 10: 433-439, 2004.

20. Jen J, Kim H, Piantadosi S, Liu ZF, Levitt RC, Sistonen P, Kinzler KW, Vogelstein B and Hamilton SR: Allelic loss of chromosome $18 \mathrm{q}$ and prognosis in colorectal cancer. N Engl J Med 331: 213-221, 1994.

21. Lanza G, Matteuzzi M, Gafá R, Orvieto E, Maestri I, Santini A and del Senno L: Chromosome 18q allelic loss and prognosis in stage II and III colon cancer. Int J Cancer 79: 390-395, 1998.

22. Marsh S, Collie Duguid ES, Li T, Liu X and McLeod HL: Ethnic variation in the thymidylate synthase enhancer region polymorphism among Caucasian and Asian populations. Genomics 58: 310-312, 1999.

23. Ogunbiyi OA, Goodfellow PJ, Herfarth K, Gagliardi G, Swanson PE, Birnbaum EH, Read TE, Fleshman JW, Kodner IJ and Moley JF: Confirmation that chromosome 18q allelic loss in colon cancer is a prognostic indicator. J Clin Oncol 16: 427-433, 1998.

24. Watanabe T, Wu TT, Catalano PJ, Ueki T, Satriano R, Haller DG, Benson AB III and Hamilton SR: Molecular predictors of survival after adjuvant chemotherapy for colon cancer. N Engl J Med 344: 1196-1206, 2001.

This work is licensed under a Creative Commons Attribution-NonCommercial-NoDerivatives 4.0 International (CC BY-NC-ND 4.0) License. 